\title{
European consumers' interest toward nutritional information on wine labeling: A cross-country analysis*
}

\author{
Azzurra Annunziata ${ }^{1}$, Eugenio Pomarici ${ }^{2}$, Riccardo Vecchio $^{3}$, and Angela Mariani ${ }^{1}$ \\ ${ }^{1}$ Department of Economic and Legal Studies, University of Naples "Parthenope", Italy \\ ${ }^{2}$ Department of Land, Environment, Agriculture and Forestry, University of Padua, Padua, Italy \\ ${ }^{3}$ Department of Agriculture, University of Naples Federico II, Italy
}

\begin{abstract}
This paper explores, through an empirical analysis European consumers' interest toward nutritional information on wine labels, examining interest, knowledge and understanding of these information. In this regard, results from direct survey with a cross-country sample of 500 wine consumers (i.e. respondents drinking at least once a month) living in Italy, France and Spain are presented and discussed. Preliminary results reveal that consumers are quite confused about the nutritional aspects of wine and tend to be interested in receiving nutritional information on wine labels. However, the interest expressed towards this kind of information differ from country to country and is influenced by other socio-demographic variables. Findings from current research should be valuable to contribute to the debate on updating international and national standards on wine labelling concerning nutrition. At the same time, the research provides a number of useful indications for policy makers in defining future development of wine nutritional labelling programs and in implementing strategies focused in enhancing efficacy and readability of labels.
\end{abstract}

\section{Introduction}

Efforts to promote healthy food consumption have risen higher on the international and national agendas; food labels are a core element to communicate nutritional information to consumers. For example, the World Health Organization recommends the provision of accurate and balanced information for consumers to enable them to make more informed and healthy choices [1].

In this regard recent developments in food legislation concerned with consumer information and health issues have created new challenges also for the wine sector particularly with reference to the new EU Food labelling Regulation 1169/2011 that in the near future may be applicable also to wine and spirits.

These changes have fostered a strong debate in the professional world and in the academic society on the efficacy and utility of this type of interventions in the wine sector. The core issue of current discussions is related to the efficacy of these initiatives to drive consumers' decisions toward more healthy and balanced choices, practically contributing to the enhancement of public health, and compensating producers' higher costs [2].

Governments have to act to protect and promote public health, but at the same time have to consider that regulatory decisions that affect the food industry can have major impacts on the economy [3].

The basic premise of including nutritional information on product labeling is that the information presented at the point of purchase or time of use will provide heightened

\footnotetext{
* This paper has been carried as part of OIV project titled "Effectiveness of health and nutritional labelling on wine: exploring current policies and consumers attitudes".
}

perceptual awareness, comprehension, and subsequent behaviour change relating to the message content [4]. At the same time providing nutrition information increases incentives for producers to create more healthful foods and aids consumers in choosing an healthier diet, which leads to lower costs from diet related illnesses [5]. However as widely recognized by literature the benefits of nutrition labelling depend on the extent to which consumers' use of nutritional labels will move food consumption towards healthier choices [6].

In literature, empirical studies have found labels to be both successful and unsuccessful in educating consumers and changing consumption behavior. At same time a large amount of studies have highlighted that label is a key source of information also for wine consumers, providing details on both intrinsic and extrinsic quality cues e.g. [7,8].

However while many studies have been conducted to analyse consumer response to nutritional labelling on other food products and the effects of nutrition label format, consumers' interest and preferences to wine nutritional labelling is rarely investigated [9-12]. Results from these studies are contradictory. Indeed even if these studies reveal the strong interest expressed by consumers to the inclusion of nutritional information on wine label, others demonstrate that the same inclusion may have both potential benefits and unintended consequences [3].

Thomson et al. (2012) [12] showed that community strongly support for a range of information that could potentially be mandated on alcoholic beverages labels included nutritional information.

Also Kypri et al. (2007) [9] suggest that the majority of Australian university students in their sample want to see both ingredients and nutritional information displayed on alcoholic beverage packaging; while no more than $4 \%$ of respondents disagreed with each of these measures. 
While Bui et al. (2008) [10], in their pilot study with university students, indicate that the availability of serving Facts information significantly decreased calorie and carbohydrate evaluations for wine and, therefore, increased consumption intentions.

Based on these considerations the core objective of the current research is to provide insights regarding European consumers' interest to the provision of nutritional information on wine labels.

\section{Methodology and data collection}

The paper explores through an empirical analysis, consumer attitudes toward nutritional information on wine labels, examining interest, knowledge and understanding of these information.

In particular, the following research questions were deeply investigated:

i) are wine consumers interested in nutritional information on labels?

ii) which is the level of knowledge and understandability of these information?

iii) how do individual consumers value these information on labels?

In this regards, results from direct survey with a crosscountry sample of 500 wine consumers (i.e. respondents drinking at least once a month) living in Italy (n.180), France (n.160) and Spain (n.160) are presented and discussed. To allow comparisons among countries, the selected convenience samples are similar in most sociodemographic features.

Data collection was performed, between January and March 2015. A professional marketing company recruited all participating consumers in the three countries via e-mails. Data collected was submitted to exploratory analysis. Univariate descriptive statistics and one-way tabulation were applied to synthesize the main characteristics of each of the variables in the total sample.

Multivariate descriptive statistics with cross-tabulation and mean comparisons were performed to get a first understanding of the relationship between the nationality and different variables and enabling the joint examination of two or more variables. Chi-square test was used to test relationships found in cross-tabulations for nominal and ordinal variables; while ANOVA with $t$-test was used to determine whether there is a significant difference in the averages of interviewees from different countries.

Table 1. Socio-demographic profile of samples.

\begin{tabular}{|l|l|l|l|}
\hline & Spain & France & Italy \\
\hline Gender: female & 52 & 50 & 51 \\
\hline Age cohort: 35-44 & 22 & 23 & 21 \\
\hline Age cohort: 45-54 & 24 & 22 & 24 \\
\hline Bachelor's degree & 39 & 40 & 39 \\
\hline Medium income & 66 & 63 & 65 \\
\hline $\begin{array}{l}\text { Main responsible for } \\
\text { household food shopping }\end{array}$ & 76 & 72 & 78 \\
\hline
\end{tabular}

\section{Main results}

Chi-square tests of the null hypothesis that the groups are identical for each observed characteristics indicated that the groups are not significantly different from socio-demographics profile at the $5 \%$ significance level.

As reported in Table 1 with reference to the gender our sample is composed mainly of woman (around 50\% in all countries). Individuals in the sample are often the main responsible for household food shopping. As for the age cohort, individuals between 35 and 54 years old mainly compose the final sample. Almost $40 \%$ of sample had a bachelor's degree and more than $60 \%$ in each country stated to have a medium income.

Firstly, respondents' propensity to use nutritional information on the food label was analysed using five statements, related to individuals' general food-buying behaviour. Specifically, respondents were asked how much specific statements reflected everyday food-shopping behaviour (scale ranging from $1=$ not at all, to 5 = completely).

In particular, Table 2 shows that on average consumers of all three countries state to pay attention to nutrition labels when buying food. However, they stated to find some difficulties in understanding the information on nutritional label and they do not devote much time to read carefully the nutritional labels while shopping.

Comparing the mean scores on Table 2 Italian consumers' state greater difficulty in the interpretation of the information and use less the nutritional information to compare products.

Than respondents were asked their level of agreement regarding the implication of wine consumption on health with several statements. More than half of the sample in each country agreed with the statement that wine could affect their health if consumed in excess (52\% in Italy, $51 \%$ in Spain and 54\% in France). More than 30\% agreed with the statement that wine could have beneficial effects on health, if it is taken in limited quantities (38\% in Italy, $34 \%$ in Spain and France). On the contrary, with reference to the statement related to contributes of excessive wine consumption to overweight and obesity, significant

Table 2. Consumers' mean scores to selected statements.

\begin{tabular}{|l|l|l|l|l|l|}
\hline & Spain & France & Italy & Overall & Sig. \\
\hline $\begin{array}{l}\text { I always pay } \\
\text { attention to } \\
\text { nutritional } \\
\text { information on } \\
\text { food labelling }\end{array}$ & 3.1 & 3.3 & 3.2 & 3.2 & .339 \\
\hline $\begin{array}{l}\text { I use nutrition } \\
\text { labelling to } \\
\text { compare products }\end{array}$ & 2.7 & 2.9 & 2.4 & 2.6 & .003 \\
\hline $\begin{array}{l}\text { I devote time to } \\
\text { read carefully the } \\
\text { nutritional labels } \\
\text { while shopping }\end{array}$ & 2.2 & 2.3 & 2.5 & 2.3 & .001 \\
\hline $\begin{array}{l}\text { I find difficulties in } \\
\text { understanding the } \\
\text { nutritional label }\end{array}$ & 3.1 & 2.9 & 3.0 & 3 & .022 \\
\hline
\end{tabular}




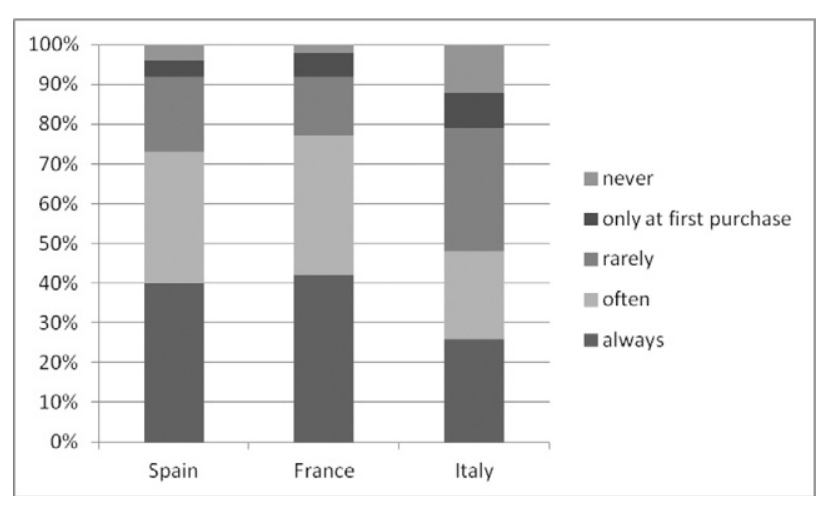

Figure 1. Reading frequencies of information on back-labels.

differences between interviews from different countries are revealed.

In particular, while French consumers completely agree with this statement in $48 \%$ of cases; Spanish and Italian consumers show a lower degree of agreement (respectively $31 \%$ and $34 \%$ ), while the percentage of those who say they slightly agree is higher in both countries (24\% in Spain and $21 \%$ in Italy) than in France.

With reference to consumers self reported reading frequencies of information on labels while choosing wine, our results show that in absolute French consumers are more careful declaring to always read these labels in 54\% of cases. While Italians appear to be less attentive to this information tool, showing a higher percentage of individuals who say they do not read them at all $(6 \%)$ or only on the first purchase $(12 \%)$.

This difference is even more pronounced when we focus on the information on the back-label. Figure 1 shows that more than $40 \%$ of French and Spanish consumers state to always read wine back-label, while for Italians this percentage drops to $26 \%$.

With reference to self-reported knowledge of wine nutritional content, our results reveal that on average respondents' state to be quite informed. Nevertheless, also in this case we can notice significant differences among countries $(p=0.002)$.

French and Spanish consumers state a higher selfreported knowledge (I know it very well for $21 \%$ and $19 \%$ of samples), compared to Italians among which prevail individuals with low self-reported (I know it poorly 48\%).

Subsequently the degree of effective knowledge of wine nutritional properties was tested by asking respondents to indicate the amount of kcal contained in a glass of $125 \mathrm{ml}$ of red wine with a medium alcoholic content (12 vol.). Moreover, we asked respondents to indicate among several alternatives proposed (alcopops, beer, wine and grappa) which alcoholic drinks contain more kcal.

Findings reported in Fig. 2 show significant differences in the level of knowledge of the amount of kcal in a glass of red wine between countries $(p=0.000)$. Particularly among Italian consumers, only $22 \%$ are aware of kcal amount in a glass of wine. Indeed most individuals tend to underestimate the content of $\mathrm{kcal}(51 \%)$ or even consider that wine has no kcal at all (12\%). Similarly, in Spain the percentage of those who tend to underestimate kcal content in wine is high $(50 \%)$, however, the correct answer

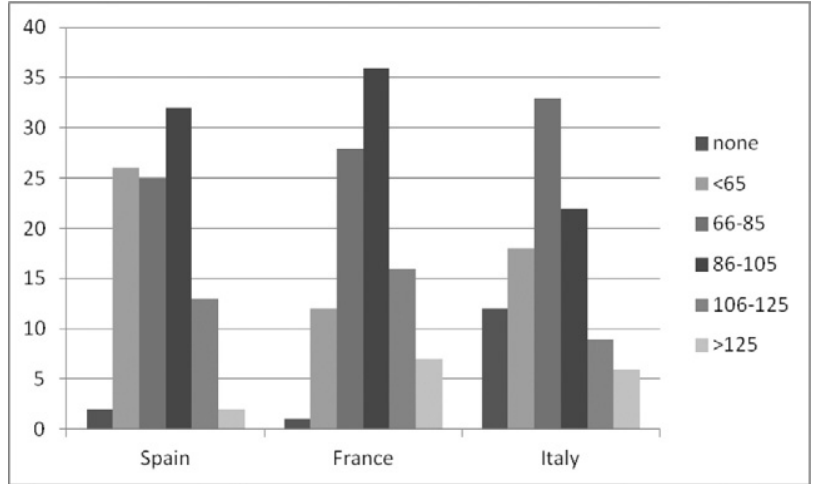

Figure 2. kcal amount in a glass of red wine.

is indicated by more than $30 \%$ of the sample. On the contrary, in France, consumers show a greater knowledge of kcal contained in a glass of wine $(36 \%)$ and in absolute tend to underestimate less the calorie content.

Also considering the selection of the drink with the highest calorie amount, findings reveal significant differences in actual knowledge shown by respondents of the examined countries. Spanish consumers are those that best identify (68\%) the drink with more calories; followed by the French $(57 \%)$ and Italians (34\%). Specifically, Italians are quite confused indicating the mug of beer in $32 \%$ of cases and the glass of wine in $10 \%$.

Subsequently we examined the interest of respondents in receiving more nutritional information on wine. In this regard, considering the total sample about $38 \%$ stated to consider useful to receive more nutritional information on wine. However, making a comparison between the responses emerged in different countries we should notice that Italian consumers are those who attribute on average greater utility to this additional information (29\% very useful and $19 \%$ very much). While French respondents attribute less utility to this information (21\% not at all useful, $28 \%$ poorly useful).

Respondents were then asked to indicate their interest on additional information on wine labels (scale ranging from $1=$ not at all, to $5=$ totally) with specific reference to the nutritional value (kcal, sugars, carbohydrates, etc.), the maximum quantity of glasses not to exceed, the amount of glasses contained in a bottle and information about possible side effects related to excessive wine consumption

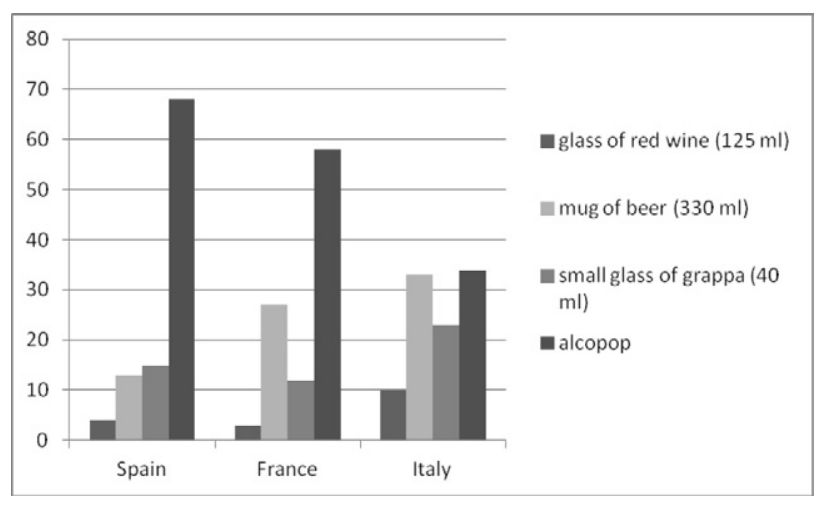

Figure 3. Indication of drink with higher amount of kcal. 
Table 3. Consumers' mean scores of additional information.

\begin{tabular}{|l|l|l|l|l|l|}
\hline & Italy & Spain & France & Overall & Sig. \\
\hline $\begin{array}{l}\text { Nutritional value } \\
\text { (kcal., sugars, } \\
\text { carbohydrates, etc.) }\end{array}$ & 3.4 & 2.9 & 2.2 & 2.9 & .000 \\
\hline $\begin{array}{l}\text { Maximum quantity } \\
\text { of glasses not to } \\
\text { exceed }\end{array}$ & 3.2 & 2.9 & 2.3 & 2.8 & .000 \\
\hline $\begin{array}{l}\text { Quantity of glasses } \\
\text { contained in a bottle }\end{array}$ & 2.5 & 2.6 & 1.9 & 2.3 & .001 \\
\hline $\begin{array}{l}\text { Information about } \\
\text { possible side effects }\end{array}$ & 3.4 & 3.6 & 3.3 & 3.4 & .009 \\
\hline
\end{tabular}

(e.g. effects of alcohol on violence, effects of taking alcohol with medicines).

Table 3 reports the mean results for each country and overall average scores. In particular, we should highlight that among the additional information consumers state a higher interest towards information on possible side effects related to excessive consumption, followed by nutritional value. Furthermore, findings, once again, underline significant differences among countries.

Italian respondents are more interested in receiving additional information on wine nutritional value and on possible side effects related to excessive consumption. Differently, French consumers assign lower interest to nutritional value information on the label, while are more concerned about possible side effects related to excessive consumption. Taking into account Spanish respondents, nutritional value information score is close to overall sample average. Whereas utility score for information about possible side effects related to excessive consumption is higher than sample mean.

Finally, we examined the effect of socio-demographic variables on consumers' interest towards nutritional and health information. Findings reveal that women and younger adults are more interested on nutritional information on wine, especially in Italy and Spain. For French consumers, instead, gender and age are not significant. Whereas other variables are statistically significant $(p<0.005)$ on consumer interest toward this information for all the three countries. In particular, presence of medical disorders that influence diet and the presence in the household of children under 16 years old.

\section{Discussion of results}

Recent changes in nutritional labelling regulation have fostered a strong debate in the professional world and in the academic society on the efficacy and utility of this type of interventions also in the wine sector. Economic theory suggests that the appropriate role for government in labeling depends on the type of information involved and the level and distribution of the costs and benefits of providing that information $[5,13,14]$.

Undoubtedly, mandatory nutritional labelling would mean significant changes for the wine sector, with huge associated increases in costs [2,3]. Therefore, its introduction requires a cautious valuation of real consumer benefits.
However, consumers' interest and preferences for wine nutritional labelling is rarely investigated and results from existing literature are contradictory.

Our findings reveal that European consumers are quite confused about the nutritional aspects of wine and tend to be interested in receiving nutritional information on wine labels. In particular, Italian and Spanish consumers seem to under estimate kcal content of wine, while French have a higher knowledge.

Moreover, interest expressed in receiving nutritional information on wine labels differ from country to country and is influenced by other socio-demographic variables. Specifically, Italian consumers are those who attribute on average greater utility to this additional information while French respondents assign lower scores.

At the same time it useful to highlight that our results report that European consumers find some difficulties in understanding current nutritional information on labels. In addition, respondents state to not devote much time reading carefully the nutritional labels while food shopping. These results are consistent with other researches on information on food labels in Europe [15-17,4] that reported nutrition labeling particularly confusing for consumers, especially due to the use of technical/scientific or numerical information.

Finally, even if our findings reveal consumers' interest towards nutritional labelling on wine several limitations suggest the need for careful consideration of labelling regulations.

\section{References}

[1] World Health Organisation. Status Report on Alcohol and Health in 35 European Countries (2013).

[2] De Almeida, A. R. (2013). New Challenges For The Wine Legislation: Allergens, Ingredients, Nutritional Declaration And Gmo-A Jurisprudence Approach. Bulletin De L'oiv, 86 (986-88), 171-180.

[3] Battaglene, T. (2014). An analysis of ingredient and nutritional labeling for wine. In BIO Web of Conferences (3, p. 03006). EDP Sciences.

[4] Grunert, K. G., \& Wills, J. M. (2007). A review of European research on consumer response to nutrition information on food labels. Journal of Public Health, 15(5), 385-399.

[5] Golan, E., Kuchler, F., Mitchell, L., Greene, C., \& Jessup, A. (2001). Economics of food labeling. Journal of Consumer Policy, 24(2), 117-184.

[6] Loureiro, M. L., Gracia, A., \& Nayga, R. M. (2006). Do consumers value nutritional labels?. European Review of Agricultural Economics, 33(2), 249-268.

[7] Lockshin, L. S., Spawton, A. L., \& Macintosh., G. (1997). Using Product, Brand, and Purchasing Involvement for Retail Segmentation. Journal of Retailing and Consumer Services, 4(3), 171-183.

[8] Thomas, A., \& Pickering, G. (2005). X-it: Gen-X and older wine drinker comparisons in New Zealand. International Journal of Wine Marketing, 17(2), 30-48.

[9] Kypri, K., McManus, A., Howat, P. M., Maycock, B. R., Hallett, J. D., \& Chikritzhs, T. N. (2007). Ingredient and nutrition information labelling of alcoholic beverages: do consumers want it?. Medical Journal of Australia, 187(11), 669-669. 
[10] Bui, M., Burton, S., Howlett, E., \& Kozup, J. (2008). What am I drinking? The effects of serving facts information on alcohol beverage containers. Journal of Consumer Affairs, 42(1), 81-99.

[11] Wright, C. A., Bruhn, C. M., Heymann, H., \& Bamforth, C. W. (2008). Beer and wine consumers' perceptions of the nutritional value of alcoholic and nonalcoholic beverages. Journal of food science, 73(1), H8-H11.

[12] Thomson, L. M., Vandenberg, B., \& Fitzgerald, J. L. (2012). An exploratory study of drinkers views of health information and warning labels on alcohol containers. Drug and alcohol review, 31(2), 240-247.

[13] Caswell, J. A., \& Padberg, D. I. (1992). Toward a more comprehensive theory of food labels. American Journal of Agricultural Economics, 460-468.
[14] Caswell, J. A., Ning, Y., Liu, F., \& Mojduszka, E. M. (2003). The impact of new labeling regulations on the use of voluntary nutrient-content and health claims by food manufacturers. Journal of Public Policy \& Marketing, 22(2), 147-158.

[15] Cowburn, G., \& Stockley, L. (2005). Consumer understanding and use of nutrition labelling: a systematic review. Public health nutrition, 8(01), 21-28.

[16] Drichoutis, A. C., Lazaridis, P., \& Nayga, R. M. (2006). Consumers' use of nutritional labels: a review of research studies and issues. Academy of Marketing Science Review, 9(9), 1-22.

[17] Drichoutis, A. C., Lazaridis, P., \& Nayga, R. M., Jr. (2005). Nutrition knowledge and consumer use of nutritional food labels. European Review of Agricultural Economics, 32(1), 93-118. 